\title{
RISCO DE QUEDAS EM IDOSOS COM DOENÇA DE PARKINSON E DEMÊNCIA DE ALZHEIMER: UM ESTUDO TRANSVERSAL
}

\author{
Christofoletti $\mathrm{G}^{1}$, Oliani MM ${ }^{1}$, Gobbi LTB $^{1}$, Gobbi $\mathrm{S}^{1}$ e Stella F ${ }^{1,2}$ \\ ${ }^{1}$ Programa de Pós-Graduação em Ciências da Motricidade, Instituto de Biociências, Universidade Estadual Paulista \\ "Júlio de Mesquita Filho" - Unesp, Rio Claro, SP - Brasil \\ ${ }^{2}$ Ambulatório de Neuropsiquiatria e Saúde Mental do Idoso, Hospital das Clínicas, Universidade Estadual de Campi- \\ nas, Campinas, SP - Brasil
}

Correspondência para: Gustavo Christofoletti, Rua Hilário da Silva Miranda, 118, Jd. Novo Chapadão, CEP 13066-300, Campinas, SP - Brasil, e-mail: gustavo_physio@yahoo.com.br

Recebido: 27/01/2006 - Revisado: 15/05/2006 - Aceito: 20/06/2006

\begin{abstract}
RESUMO
Objetivo: Comparar o risco de quedas entre idosos com doença de Parkinson (DP), demência de Alzheimer (DA) e saudáveis (controle). Além disso, pretendeu-se analisar as relações do risco de quedas com declínio cognitivo e com nível de atividade física. Método: vinte idosos, sendo sete com DP (69,57 $\pm 2,40$ anos), seis com DA (77,5 $\pm 2,32$ anos) e sete saudáveis (74,71 \pm 2,58 anos), foram avaliados por meio dos seguintes instrumentos: Escala de Equilíbrio Funcional de Berg (EEFB), Timed Up and Go test (TUG), Mini-Exame do Estado Mental (MEEM) e Questionário Baecke Modificado para Idosos (QBMI). Resultados: O teste de Kruskal-Wallis apontou diferença significativa entre os grupos, tanto em relação à EEFB (KW=9,67, $\mathrm{p}<0,01)$, quanto em relação ao TUG (KW=9,14, p<0,01, para tempo despendido, e $K W=10,04, p<0,01$, para número de passos). A “análise aos pares" do teste post-hoc de Bonferroni apontou comprometimento maior do equilíbrio no grupo DA, no qual também foram observados menores valores no MEEM. O grupo DP foi caracterizado por apresentar um maior nível de atividade física. A análise de correlação de Spearman apontou correlação baixa entre MEEM e EEFB ( $\left.r_{s}=0,59\right)$; entre MEEM e TUG ( $r_{s}=-0,62$ e $\left.r_{s}=-0,52\right)$; entre QBMI e EEFB ( $\left.r_{s}=0,54\right)$; e entre QBMI e TUG ( $r_{s}=-0,39$ e $\left.r_{s}=-0,42\right)$. Conclusões: As baixas correlações observadas devem ser analisadas com cautela, pois tais variáveis sofrem influência de múltiplos fatores. No grupo DA, o declínio cognitivo pode estar relacionado ao maior risco de quedas e, no grupo DP, a atividade física pode ter auxiliado a manter um risco de quedas próximo ao grupo controle.
\end{abstract}

Palavras-chave: risco de quedas, doença de Parkinson, demência de Alzheimer.

\section{ABSTRACT \\ Risk of Falls Among Elderly People with Parkinson's Disease and Alzheimer's Dementia: A Cross-Sectional Study}

Objective: To compare the risk of falls among elderly people with Parkinson's disease (PD) and Alzheimer's dementia (AD) and among healthy peers (controls). In addition, the aim was to analyze relationships between risk of falls and cognitive decay and between risk of falls and physical activity. Method: Twenty subjects were assessed: seven with PD (69.57 \pm 2.40 years), six with $\mathrm{AD}(77.5 \pm 2.32$ years) and seven healthy peers $(74.71 \pm 2.58$ years $)$. The following instruments were used: Berg Balance Scale (BBS), Timed Up and Go test (TUG), Mini-Mental State Examination (MMSE) and Modified Baecke Questionnaire for Older Adults (MBQOA). Results: The Kruskal-Wallis (KW) test indicated significant differences between the groups, relating to BBS (KW = 9.67, $\mathrm{p}<0.01)$ and TUG (KW = 9.14, $\mathrm{p}<0.01$, for time expended, and KW = 10.04, $\mathrm{p}<0.01$, for number of steps). Bonferroni post-hoc pairs analysis showed that balance was highly compromised in the AD group, such that lower MMSE values were observed. The PD group was characterized by presenting higher levels of physical activity. Spearman's test produced low correlations between MMSE and BBS ( $\left.r_{s}=0.59\right)$; MMSE and TUG ( $r_{s}=-0.52$ and $\left.r_{s}=-0.62\right)$; MBQOA and BBS $\left(r_{s}=0.54\right)$; and MBQOA and TUG $\left(r_{s}=-0.39\right.$ and $\left.r_{s}=-0.42\right)$. Conclusion: These low correlations must be analyzed carefully, because these variables are influenced by multiple factors. In the AD group, the cognitive decay can be positively associated with greater risk of falls. In the PD group, physical activity may have assisted in maintaining the risk of falls at similar levels to the control group.

Key words: risk of falls; Parkinson’s disease; Alzheimer’s dementia. 


\section{INTRODUÇÃO}

O fato de os seres humanos serem bípedes e de se locomoverem com fases de apoio unipodal (deambulação), sem nenhum contato (corrida), ou permanecer parado com o contato de ambos os pés (ortostatismo), cria um desafio adaptativo aos sistemas que controlam o equilíbrio, tornandose necessárias informações contínuas sobre a posição e o movimento de todas as partes do corpo $^{1,2}$. O controle da posição do corpo no espaço, como forma de promover estabilidade e orientação, é denominado de controle postural. A estabilidade postural é alcançada através do repouso (equilíbrio estático), do movimento estável (equilíbrio dinâmico) ou pela recuperação da postura estática (equilíbrio recuperado). Nessas situações, o centro de massa do corpo deve estar projetado dentro dos limites da base de apoio e se faz fundamental a integração das informações sensoriais com os sistemas neuromusculares ${ }^{3}$.

A alta incidência e prevalência de quedas em idosos decorrem de alterações intrínsecas e extrínsecas ${ }^{4,5,6}$. Dentre os fatores intrínsecos, destacam-se as alterações sensóriomotoras inerentes ao processo de envelhecimento (alterações visuais, parestesias, paresias, diminuição de flexibilidade e de mobilidade e declínio cognitivo); os fatores extrínsecos, fortemente associados às dificuldades propiciadas pelo ambiente (buracos, escadas e terrenos irregulares), constituem também grande risco de quedas ${ }^{6,7,8,9}$.

Alterações fisiopatológicas características de algumas doenças também são responsáveis por quedas na população idosa. Dentre elas, destacam-se as síndromes cerebelares, as vestibulopatias, os distúrbios osteomioarticulares e as patologias neurodegenerativas ${ }^{10}$. Nesse último grupo, encontram-se a doença de Parkinson (DP) e a demência de Alzheimer (DA), dentre outras.

A DP consiste em uma patologia crônica e progressiva, caracterizada pela degeneração de neurônios dopaminérgicos da pars compacta da substantia nigra mesencefálica ${ }^{11}$. Conseqüente à atrofia e à degeneração dos núcleos da base, o paciente com DP apresenta distúrbios motores freqüentes, como tremor, rigidez, bradicinesia, freezing e festinação $0^{12}$. Dificuldades no equilíbrio são comuns em sujeitos com essa enfermidade.

Pahapill e Lozano ${ }^{13}$ atribuem as alterações nas reações de equilíbrio de pacientes com DP à degeneração de neurônios glutamatérgicos dos núcleos pedunculopontinos, lembrando que a pars dissipatus desses núcleos recebe aferências da medula espinhal e dos núcleos da base e projeta conexões eferentes ao cerebelo e à medula espinhal. No cerebelo, informações corticais da área de associação são transmitidas, formando-se, assim, a via córtico-ponto-cerebelar relacionada com o planejamento do movimento. A atrofia e a degeneração dos núcleos da base geram um padrão inibitório exacerbado, fazendo com que o paciente com DP encontre dificuldade em modular as estratégias de equilíbrio. Colnat-Coulbois et al. ${ }^{14}$, no entanto, atribuem as dificuldades no equilíbrio não somente às alterações motoras da doença. Segundo esses autores, o indivíduo com DP se encontra em conflito constante de processamento sensitivo central, pois entra em contato com informações visuais e somatossensoriais íntegras e com reações vestíbulo-galvânicas exacerbadas.

Por outro lado, a DA consiste em uma patologia crônica e progressiva, caracterizada por declínio da memória recente e de outras funções cognitivas decorrente da depleção da acetilcolina nos núcleos basais de Meynert e da atrofia do lobo temporal, principalmente da formação hipocampal ${ }^{15}$. Diferente da DP, que consiste em um distúrbio sobretudo motor, a DA é caracterizada por alterações cognitivas inerentes à atrofia cerebral e à formação de placas amilóides e emaranhados neurofibrilares ${ }^{16}$. Alterações nas reações de equilíbrio são comuns nos estágios avançados da doença ${ }^{10,17}$.

Kato et al. ${ }^{10}$ relatam que, em estágios leves, o risco de queda do indivíduo com DA equipara-se ao risco de quedas de idosos saudáveis. Entretanto, processos motores regulados por mecanismos corticais complexos, como sentar-se e levantar-se da cadeira, podem estar afetados nos estágios leve e moderado da DA, predispondo o paciente ao risco de quedas ${ }^{18}$. No estágio avançado, no entanto, o aumento do risco de quedas está relacionado ao provável comprometimento visual e às alterações cognitivas ${ }^{17,19}$. Carmeli et al..$^{19}$ observaram que o risco de quedas é alto em idosos com déficit cognitivo, pois, geralmente, esse se encontra associado à negligência, exclusão social e sintomas depressivos. Esses fatores, por sua vez, contribuem para reduzir o desempenho em atividade física e aumentar a fraqueza muscular global.

A fisioterapia e a atividade física constituem ferramentas importantes para prevenir e/ou minimizar déficits de equilíbrio em idosos ${ }^{20,21}$. Benefícios da intervenção motora foram constatados nos sistemas cardiovascular, neuromuscular e sensorial $^{7,22}$. Alguns estudos comprovam, ainda, efeitos da atividade física sobre as funções cognitivas ${ }^{23}$. Diante do exposto, este estudo teve como objetivo comparar o risco de quedas entre idosos com DP, DA e idosos saudáveis. Além disso, pretendeu-se analisar as relações do risco de quedas com o declínio cognitivo e com o nível de atividade física.

\section{MÉTODOS}

Este estudo envolveu idosos com DP, DA e saudáveis (grupo controle), residentes na cidade de Rio Claro - SP. O grupo controle foi constituído por idosos com idade igual ou superior a 60 anos e que tinham preservadas suas habilidades de marcha e equilíbrio. Foram excluídos, desse grupo, sujeitos com condições clínicas que interferissem no desempenho motor e no equilíbrio, como amaurose, síndromes vertiginosas (associadas ou não ao uso de medicamentos), claudicações de diferentes etiologias e distúrbios do movimento relacionados com declínio cognitivo. O grupo de pacientes, também com 60 anos ou mais, foi constituído de sujeitos 
com DP em estágio igual ou superior a 2 na Escala de HoehnYarh $^{24}$ e de pacientes com DA moderada, segundo o Escore Clínico de Demência ${ }^{25}$. Os indivíduos com DP foram avaliados na fase on da medicação. Este estudo não apresentou perdas amostrais, qualquer que fosse o grupo analisado.

Para a avaliação do equilíbrio, foram utilizados a Escala de Equilíbrio Funcional de Berg (EEFB) ${ }^{26,27}$ e o teste Timed Up and Go (TUG) ${ }^{28}$. A EEFB é uma escala composta por 14 itens envolvendo tarefas funcionais específicas em diferentes bases de apoio. O escore varia entre 0 e 56, com pontuações inferiores caracterizando um maior risco de quedas. O TUG avalia mobilidade funcional básica. Nele é analisado o tempo gasto pelo indivíduo para se levantar de uma cadeira com braços, andar por uma distância de 3 metros e retornar à cadeira, bem como o número de passos necessários para a execução da atividade. Maiores valores de tempo e número de passos representam maior risco de quedas.

Para avaliar as funções cognitivas foi aplicado o Mini-Exame do Estado Mental (MEEM) ${ }^{29}$. Esse instrumento compõe-se de sete categorias, cada uma delas planejada com o objetivo de avaliar funções cognitivas específicas. São elas: orientação para tempo, orientação para local, registro de três palavras, atenção e cálculo, recordação das três palavras, linguagem e praxia visuo-construtiva. O escore do MEEM varia de 0 a 30 pontos, sendo que valores mais baixos apontam para possível déficit cognitivo. Como o teste sofre influência da escolaridade, valores de referência foram propostos com o objetivo de identificar sujeitos com possíveis déficits cognitivos. Brucki et al. ${ }^{30}$ estudaram uma amostra brasileira e sugeriram os seguintes valores para estudos em nosso meio: para analfabetos, 20 pontos; de 1 a 4 anos de escolaridade, 25; de 5 a 8 anos, 26,5; de 9 a 11 anos, 28; e, para indivíduos com escolaridade superior a 11 anos, 29 pontos.

Com o objetivo de quantificar o nível de atividade física, utilizou-se o Questionário Baecke Modificado para Idosos $(\mathrm{QBMI})^{31}$. O QBMI avalia o nível de atividade física em três domínios específicos: tarefas realizadas em casa, atividades esportivas e atividades de lazer. Os escores são obtidos através de perguntas específicas e pela relação entre tipo, freqüência e intensidade da atividade. Escores menores, nesse instrumento, representam um menor nível de atividade física realizado pelo sujeito.

A análise estatística consistiu de: a) teste de KruskalWallis, para se comparar o risco de quedas entre os grupos; b) teste post-hoc de Bonferroni, para realizar "comparação aos pares”; c) coeficiente de correlação de Spearman, para analisar as relações entre nível de atividade física e risco de quedas e entre declínio cognitivo e risco de quedas. Admitiuse, a priori, nível de significância de $5 \%(\mathrm{p}<0,05)$.

O presente trabalho foi aprovado pelo Comitê de Ética em Pesquisa (protocolo no. 4494). Os participantes assinaram um Termo de Consentimento Livre e Esclarecido baseado na resolução 196/96 do Ministério da Saúde. Especificamente nos casos de sujeitos com DA, nos quais o déficit cognitivo é característico da doença, o Termo de Consentimento Livre e Esclarecido foi assinado pelo participante e pelo familiar responsável.

\section{RESULTADOS}

Este estudo envolveu vinte idosos, sendo sete com DP $(69,57 \pm 2,40$ anos, amplitude 62 a 80$)$; seis com DA (77,5 $\pm 2,32$ anos, amplitude 69 a 89) e sete idosos saudáveis $(74,71$ $\pm 2,58$ anos, amplitude 65 a 85). Dados referentes à idade, escolaridade, equilíbrio funcional, mobilidade, funções cognitivas e informações sobre atividade física são apresentados na Tabela 1.

Tabela 1. Características dos grupos quanto à idade, escolaridade, risco de quedas, funções cognitivas e nível de atividade física.

\begin{tabular}{lccc}
\hline \multicolumn{1}{c}{ Grupos } & Parkinson & Alzheimer & Controle \\
\hline Idade (anos) & $69,57 \pm 2,40$ & $77,50 \pm 2,32$ & $74,71 \pm 2,58$ \\
Escolaridade (anos) & $9,28 \pm 1,57$ & $2,67 \pm 0,67$ & $5,00 \pm 2,00$ \\
EEFB (pontos) & $50,85 \pm 1,83$ & $39,34 \pm 3,18$ & $51,14 \pm 1,14$ \\
TUG (seg) & $9,16 \pm 1,61$ & $22,09 \pm 7,13$ & $6,27 \pm 0,31$ \\
TUG (passos) & $15,14 \pm 1,70$ & $26,34 \pm 5,12$ & $12,71 \pm 0,56$ \\
MEEM (pontos) & $28,00 \pm 0,78$ & $13,67 \pm 1,72$ & $26,00 \pm 1,38$ \\
QBMI (pontos) & $5,48 \pm 0,48$ & $2,59 \pm 0,78$ & $2,72 \pm 0,53$
\end{tabular}

EEFB: Escala de Equilíbrio Funcional de Berg; TUG: Timed Up and Go test; MEEM: Mini-Exame do Estado Mental; QBMI: Questionário Baecke Modificado para Idosos.

O teste de Kruskal-Wallis apontou diferença significativa entre os grupos, tanto em relação à EEFB $(K W=9,67, p<0,01)$ quanto em relação ao TUG (KW=9,14, p<0,01, para tempo despendido, e KW=10,04, $\mathrm{p}<0,01$, para número de passos). O teste post-hoc de Bonferroni apontou pior comprometimento do equilíbrio no grupo DA, seguido pelos grupos DP e controle. Não foi observada diferença significativa entre a idade dos sujeitos (KW=3,94, p>0,05), indicando que tal variável não influenciou os resultados dos grupos. Em relação ao índice de correlação de Spearman, foram observadas baixas correlações entre MEEM e EEFB $\left(r_{s}=0,59\right)$; entre MEEM e TUG ( $r_{s}=-0,52$, para tempo despendido, e $r_{s}=-0,62$, para número de passos); entre QBMI e $\operatorname{EEFB}\left(\mathrm{r}_{\mathrm{s}}=0,54\right)$ e; entre QBMI e TUG $\left(r_{s}=-0,39\right.$ para tempo despendido e $r_{s}=-0,42$ para número de passos). 


\section{DISCUSSÃO}

Os resultados encontrados por este estudo apresentaram diferenças em relação ao risco de quedas nos três grupos. Por meio do teste post-hoc de Bonferroni, observou-se um maior risco de quedas no grupo com DA, seguido pelos grupos DP e controle. Uma análise detalhada sobre os fatores que levam sujeitos com DA a apresentarem um maior risco de quedas do que sujeitos com DP é de fundamental importância para a prática clínica, pois pode sugerir mudanças de objetivos e condutas em ambas as patologias.

Estudos realizados por Cress et al. ${ }^{22}$ apontaram o declínio cognitivo como uma variável que influencia diretamente o risco de quedas em idosos. As baixas correlações encontradas entre MEEM e EEFB e entre MEEM e TUG devem ser analisadas com cautela. Como explicado por Samson et al. ${ }^{4}$ e Tinetti et al. ${ }^{6}$, o risco de quedas é influenciado por diversos fatores, como alterações visuais, paresias, parestesias, diminuição de flexibilidade e mobilidade. $\mathrm{O}$ fato da correlação entre declínio cognitivo e risco de quedas ter apresentado índice de determinação entre 15\% e 38\% indica que tal variável está associada ao aumento do risco de quedas em idosos e contribui para isso de maneira importante.

Ainda em relação às funções cognitivas, nos participantes com DA foram constatados valores baixos no MEEM em relação aos escores de referência propostos por Brucki et al. ${ }^{30}$. Comparando-se o risco de quedas nos grupos DP e controle (ambos sem constatação de declínio cognitivo) com os valores do grupo DA, conclui-se que o declínio cognitivo pode ter aumentado o risco de quedas na DA.

Gillespie et al. ${ }^{20}$ e Lun et al. ${ }^{21}$ apontaram os efeitos da atividade física como forma de prevenir e/ou minimizar o risco de quedas em idosos. Como discutido anteriormente, as baixas correlações entre QBMI e EEFB e entre QBMI e TUG não devem ser analisadas como um resultado contrário a esses estudos. Quando observado especificamente o grupo com DP, caracterizado por apresentar maiores índices no QBMI, conclui-se que a atividade física pode ter contribuído para minimizar as alterações de equilíbrio, fazendo com que os resultados encontrados no EEFB e no TUG fossem muito próximos aos valores do grupo controle. Em relação aos participantes com DA, qualquer argüição sobre os efeitos da atividade física deve ser analisada cautelosamente; pois, além deste grupo apresentar valores baixos no QBMI, há, como visto anteriormente, a influência do declínio cognitivo sobre o risco de quedas.

Na interpretação dos resultados encontrados, deve ser levado em conta que os participantes com DP e DA encontravam-se em graus moderados em ambas as enfermidades. Conquanto possa ser argüido sobre o pequeno tamanho amostral, há que se considerar a dificuldade de recrutamento dos sujeitos, bem como o fato de ambas as patologias apresentarem um diagnóstico complexo, exigindo a integração da avaliação clínica com exames laboratoriais e de neuroimagem, nem sempre disponíveis. Na DA, por exemplo, o diagnóstico definitivo é realizado através da autópsia cerebral. Em vida, a utilização de exames de neuroimagem estrutural (volumetria do hipocampo, por exemplo) e os de neuroimagem funcional (espectroscopia por ressonância magnética) auxiliam no diagnóstico mais próximo do correto. Já na DP, a dificuldade do diagnóstico está em diferenciar DP idiopática das síndromes parkinsonianas secundárias ao comprometimento cerebral.

Em relação aos testes que avaliam o risco de quedas, muitos deles apresentam pouca preditividade em amostras de indivíduos independentes e residentes na comunidade ${ }^{32,33}$, sendo recomendada a aplicação de dois ou mais testes, visando diminuir a probabilidade de se obter falsos positivos e negativos. Em conseqüência, julgou-se oportuno utilizar simultaneamente a EEFB e o TUG, pois apresentam alto índice de correlação $(r=-0,91)^{26}$.

Em conclusão, na amostra analisada, os participantes com DA, afetados por um distúrbio eminentemente cognitivo, apresentaram um maior risco de quedas que pacientes com DP, uma patologia com sintomatologia primordialmente motora.

Em termos de intervenção profissional, a interpretação dos resultados encontrados permite sugerir que, em indivíduos com DP ou DA, medidas de prevenção, proteção e reabilitação devem incluir estimulação motora e cognitiva. Além disso, os achados deste estudo contribuem para estimular outros estudos que visem a analisar os mecanismos pelos quais o declínio cognitivo e o nível de atividade física, em ambas as patologias, interferem no risco de quedas.

Agradecimentos: CAPES, LAFE e LEPLO (Unesp - IB - Rio Claro); FINEP; FNS-MS; FUNDUNESP; PROEX-UNESP.

\section{REFERÊNCIAS BIBLIOGRÁFICAS}

1. Winter DA. Human balance and posture control during standing and walking. Gait Posture. 1995; 3(4): 193-214.

2. Kelly JP. The sense of balance. In: Kandel ER, Schwartz JH, Jessel TM, editores. Principles of Neural Science. $3^{\text {a }}$ ed. Norwalk: Appleton \& Lange; 1991. p. 500-511.

3. Schumway-Cook A, Woollacott MH. Controle postural. In: Schumway-Cook A, Woollacott MH, editores. Controle Motor: teoria e aplicações práticas. 2ª ed. Barueri: Manole; 2003. p.153-178.

4. Samson MM, Meeuwsen IBAE, Crowe A, Dessens JAG, Duursma SA, Verharr HJJ. Relationships between physical performance measures: age, height and body weight in healthy adults. Age Ageing. 2000; (29): 235-42.

5. Whitney SL, Poole JI, Cass SP. A review of balance instruments for older adults. Am J Occup Ther. 1998; 52(8): 666-71. 
6. Tinetti ME, Inouye SK, Gill TM, Doucette JT. Shared risk factors for falls, incontinence and functional dependence: unifying the approach to geriatric syndromes. JAMA. 1995; 273(17): 1348-53.

7. Miyasike-Da-Silva V, Gonçalves CT, Silva JJ, Gobbi LTB. Mobilidade de idosos em ambiente doméstico: efeitos de um programa de treinamento específico. Rev Bras Ativ Fís Saúde. 2003; 8(1): 5-19.

8. Miyasike-Da-Silva V, Gobbi LTB. Percepção de dificuldade e comportamento locomotor de idosos ao descer degraus de ônibus. Motricidade (Portuguese Journal of Human Performance Studies). 2005; 1(2): 96-105.

9. Miyasike-Da-Silva V, Gobbi LTB. Locomotion during climbing downstairs of two different heights in young and older adults. In: 6th World Congress on Aging and Physical Activity: Proceedings from the 6th World Congress on Aging and Physical Activity; 2004; London. Ontario: Canadian Center for Activity and Aging; 2005. p. 35-7.

10. Kato EM, Radanovic M, Smid J, Caramelli P, Brucki SM, Nitrini R. Evaluation of the balance control in Alzheimer disease. Arq Neuropsiquiatr. 2005; 63(2): S110.

11. Brusse KJ, Zimdars S, Zalewski KR, Steffen TM. Testing functional performance in people with Parkinson Disease. Phys Ther. 2005; 85(2): 134-41.

12. Lim LIIK, van Wegen EEH, Goede CJT, Jones D, Rochester L, Hetherington V, et al. Measuring gait and gait-related activities in Parkinson's patients own home environment: a reliability, responsiveness and feasibility study. Parkinsonism Relat Disord. 2005; (11): 19-24.

13. Pahapill PA, Lozano AM. The pedonculopontine nucleus and Parkinson’s disease. Brain. 2000; 123(Pt9); 1767-83.

14. Colbat-Coulbois S, Gauchard GC, Maillard L, Barroche G, Vespignani $\mathrm{H}$, Auque J, et al. Bilateral subthalamic nucleus stimulation improves balance control in Parkinson's disease. J Neurol Neurosurg Psychiatry. 2005; (75): 780-7.

15. Petersen RC, Jack CR, Xu JC, Waring SC, O’Brien PC, Smith GE, et al. Memory and MRI-based hipocampal volumes in aging and AD. Neurology. 2000; 54(3): 581-7.

16. Terada S, Ishizu H, Ishiguro K, Tanabe Y, Itoh N, Yasutake K, et al. Exon 3 insert of tau protein in neurodegenerative disease. Acta Neuropatol. 2005; 110(1): 12-8.

17. Chong RK, Horak FB, Frank J, Kaye J. Sensory organization for balance: specific deficits in Alzheimer's but not in Parkinson's disease. J Gerontol. 1999; 54(3): 122-8.

18. Manckoundia P, Mourey F, Pfitzenmeyer P, Papaxanthis C. Comparison of motor strategies in sit-to-stand and back-to-sit motions between healthy and Alzheimer's disease elderly subjects. Neuroscience. 2006; (137): 385-92.

19. Carmeli E, Zinger-Vaknin T, Morad M, Merrick J. Can physical training have an effect on well-being in adults with mild intellectual disability? Mech Ageing Dev. 2005; (126): 299-304.
20. Gillespie LD, Gillespie WJ, Robertson MC, Cumming RG, Rowe BH. Interventions for preventing falls in elderly people (Cochrane Review). In: The Cochrane Library, Issue 1, 2006. Oxford: Update Software.

21. Lun V, Pullan N, Labelle N, Adams C, Suchowersky O. Comparison of the effects of a self-supervised home exercise program with a physiotherapist-supervised exercise program on the motor symptoms of Parkinson's disease. Mov Disord. 2005; 20(8): 971-5.

22. Cress ME, Buchner DV, Prohaska T, Rimmer J, Brown M, Macera C, et al. Physical activity programs and behavior counseling in older adult population. Med Sci Sports Exerc. 2004; 36(11): 1997-2003.

23. Glaesmer G, Künstler J, Reuter W. Verbesserung von grund legenden Alltagsfunktionen, Mobilität und kognitiver Leistungsfähigkeit durch Behandlung in einer geriatrischen Tagesklinik. J Gerontol Geriatr. 2003; (36): 475-483.

24. Hoehn MM,Yahr MD. Parkinsonism: onset, progression and mortality. Neurology. 1967; 17(5): 427-42.

25. Hughes CP, Berg L, Danziger WL, Coben LA, Martin RL. A new clinical scale for the staging of dementia. Br j Psychiatr. 1982; (140): 566-72.

26. Berg KO, Wood-Dauphinée SL, Williams JI, Maki B. Measuring balance in the elderly: validation of an instrument. Can J Public Health. 1992; (83): S7-11.

27. Myiamoto ST, Lombardi JI, Berg KO, Ramos IR, Natour J. Braziliam version of the Berg Balance Scale. Braz J Med Biol Res. 2004; 37(8): 1411-21.

28. Podsiadlo D, Richardson S. The "Timed Up and Go": a test of basic functional mobility for frail elderly persons. J Am Geriatr Soc. 1991; (39): 142-8.

29. Folstein MF, Folstein SE, Mchugh PR. Mini-Mental State: a practical method for grading the cognitive state of patients for the clinician. J Psychiatr Res. 1975; 12(3): 198.

30. Brucki SMD, Nitrini R, Caramelli P, Bertolucci PHF, Okamoto IH. Sugestões para o uso do mini-exame do estado mental no Brasil. Arq Neuropsiquiatr. 2003; 61(3B): 777-81.

31. Voorrips LEA, Ravelli ACJ, Dongelmans PCA, Deurenberg P, Van Staveren WA. A physical activity questionnaire for the elderly. Med Sci Sports Exerc. 1991; (12): 974-9.

32. Boulgarides LK, McGinty SM, Willet JA, Barnes CW. Use of clinical impairment based tests to predict falls by communitydwelling older adults. Phys Ther. 2003; 83(4): 328-39.

33. Pérennou D, Decavel P, Manckoundia P, Penven Y, Mourey F, Launay F, et al. Évaluation de l'équilibre en pathologie neurologique et gériatrique. Ann Readapt Med Phys. 2005; (48): 317-35. 